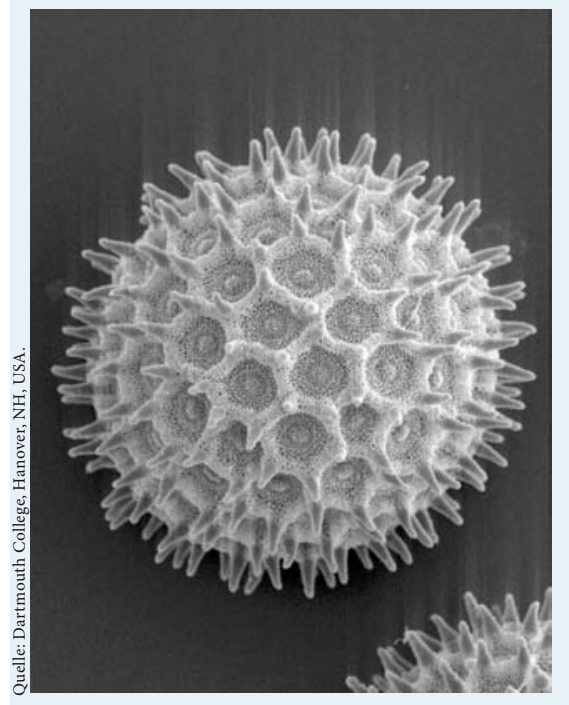

\section{Heuschnupfen und andere Allergien}

Wenn ab dem Frühjahr die Bäume ausschlagen, die Gräser blühen und die Pollen fliegen, beginnt für die Hausärzte und Hausärztinnen die Zeit, in der sich die Allergiker in der Praxis die Klinke in die Hand geben. Im Expertengespräch ab Seite 75 ist zu lesen, wie Vertreter von Homöopathie, Traditioneller Chinesischer Medizin und Phytotherapie Allergien behandeln. Neue Studien belegen, dass pasteurisierte Milch eine wichtige Rolle bei der Zunahme von Allergien in den letzten Jahrzehnten spielt und dass unbehandelte Milch aus bislang unbekannten Gründen vor innen schützt (siehe nachfolgenden Beitrag). Und mehr über die Ergebnisse einer Schweizer PhaseI-Studie mit einem rekombinanten Allergen zur Hyposensibilisierung wird auf Seite 80 geschildert.

\title{
Die Milch macht das Immunsystem munter
}

In einem Forschungsprogramm mit Schweizer Beteiligung sind Wissenschafter den Gründen für die Zunahme von Allergien auf die Spur gekommen. Die industriell verarbeitete Milch schützt nicht mehr vor Allergien.

Dass die Münchner Professorin und Kinderärztin Erika von Mutius in den letzten Jahren ziemlich viel über Milch, ihre Eigenschaften und ihre industrielle Verarbeitung gelernt hat, ist einem Forschungsprogramm der Europäischen Union zu verdanken. Erika von Mutius von der Universität München ist federführend am Forschungsprogramm «Forallvent» beteiligt gewesen. Das hatte die Aufgabe, möglichst umfassend Informationen zusammenzutragen, weshalb es in den letzten 100 Jahren zu einer starken $\mathrm{Zu}$ nahme an Allergien und Asthma gekommen ist. Tatsächlich galt in der Mitte des 19. Jahrhunderts der Heuschnupfen als fast «adelige Krankheit» und als eine Erscheinung, die nur sehr selten bei den ungebildeten Ständen vorkomme, wie der englische Arzt Charles Blackley 1873 meinte.

\section{Zunahme von Allergien}

Seitdem hat sich die Lage dramatisch verändert. Heute leidet in Westeuropa etwa ein Viertel der Bevölkerung an Heuschnupfen. Die Schweiz liegt etwa im Durchschnitt: Knapp über zwei Millionen Patienten mit Allergien gibt es hierzulande. Die Zunahme von Allergien und auch von Asthma wird von Experten auf die veränderten Umweltbedingungen zurückgeführt - doch welche das genau sind, ist unklar. In den neuen Forschungsprogrammen «Gabriel» und «Efraim», die derzeit laufen, sollen die
Ursachen der Allergenwelle aufgeklärt werden.

«Dabei könnte die Milch eine wichtige Rolle spielen», sagt Erika von Mutius. Einige Forschungsergebnisse deuten darauf hin, dass die modernen Methoden, die Milch zu verarbeiten, ihr die Schutzwirkung vor Allergenen geraubt hat. «Um die möglichen Konsequenzen zu verstehen, mussten wir uns als Mediziner mit den verschiedenen Aspekten der Milch und ihrer Produktion beschäftigen - von der Zusammensetzung bis zur Verarbeitung.» Bei den Symposien von «Forallvent» trafen sich etwa Epidemiologen, Kinderärzte und Allergologen mit Lebensmitteltechnikern, um voneinander zu lernen.

\section{Schwierige Forschungslage}

Die Entstehung von Allergien ist komplex. Zum einen können sich hinter ähnlichen Symptomen, wie etwa bei Urtikaria, unterschiedliche Ursachen verbergen. Zum anderen spielen bei der Entstehung von Allergien Fragen bezüglich Phänotyp, Zeitpunkt, Umwelt und der genetischen Disposition eine wichtige Rolle. In der Vergangenheit hat eine Reihe von Studien gezeigt, dass z.B. der Effekt der Exposition mit einem Allergen sehr stark vom Zeitpunkt im Leben des Betroffenen abhängen kann. Heuschnupfen kann im Alter erst auftauchen oder auch wieder verschwinden. Bei einer solch komplexen Ausgangslage ver-

\section{KARGER}

Fax +49761 4520714

E-Mail Information@Karger.de

www.karger.com 
spricht nur ein Forschungsansatz Erfolg, der mit einem ganzheitlichen Blick das Thema Allergien und ihre Entstehung beleuchtet. Längst ist klar, dass einfache Erklärungen nicht zum Ziel kommen. In den letzten zehn Jahren ist es allerdings gelungen, die wichtigsten Gründe für die Entstehung von Allergien zu identifizieren.

\section{Umweltverschmutzung scheidet aus}

Die Zunahme der Allergien in den letzten 100 Jahren ist lange Zeit aufdie Verschlechterung der Umweltbedingungen in den Industrienationen zurückgeführt worden. Die Belastung etwa von Wasser und Luft, die Verbreitung neuer Stoffe - von Latex über Herbizide bis zu den Weichmachern in Plastik - sollten dem Organismus so zusetzen, dass er mit einer Überreaktion des Immunsystems schon auf sonst harmlose Allergene antwortet.

Im Ostteil Deutschlands gab es zwar weniger Allergiker als im Westen, jedoch war die Umwelt dafür stärker belastet. Nach der Wiedervereinigung wurde die Luft sauberer, aber die Prävalenz etwa von Heuschnupfen und anderen Allergien stieg an. Der Grund hierfür scheint zu sein, dass in der damaligen DDR durch die frühere und häufigere Gemeinschaftsunterbringung von Kindern vermehrt
Infekte ausgelöst wurden, die das Immunsystem der Betroffenen ausreichend auslasteten, sodass keine weiteren Allergien entstehen konnten.

Viele Mediziner suchen in der Hygiene-Hypothese eine Erklärung für das Hochschnellen der Allergikerquote. Sie wurde vor 20 Jahren von David P. Strachan im British Medical Journal vorgeschlagen. Sie ging von der Beobachtung aus, dass Kinder, die mit grösseren Geschwistern aufwachsen, weniger häufig Heuschnupfen und Ekzeme haben. Durch die häufige Exposition durch Keime der Grösseren seien sie besser geschützt als Einzelkinder oder Kinder aus kleineren Familien.

In den letzten zwei Jahrzehnten ist die Hygiene-Hypothese in einer ganzen Reihe von epidemiologischen und immunologischen Studien untersucht und durch deren Ergebnisse erweitert worden. Weil die Menschen in den modernen Industriegesellschaften immer seltener mit nicht pathogenen Keimen in Berührung kommen, scheint ihr Immunsystem besonders sensibel zu werden.

Mit immunologischen Daten lässt sich die Hygiene-Hypothese stützen: Allergien werden durch eine Th2modulierte Reaktion hervorgerufen. Bakterien und Viren, die in den Körper eindringen, lösen eine durch Th1 gesteuerte Immunantwort aus, die
Abb. 1. Elektronenmikroskopaufnahme der Pollen von Oenothera fruticosa (Sonnenwende). Quelle: Louisa Howard, Dartmouth Electron Microscope Facility, Dartmouth College, Hanover, $\mathrm{NH}$, USA.

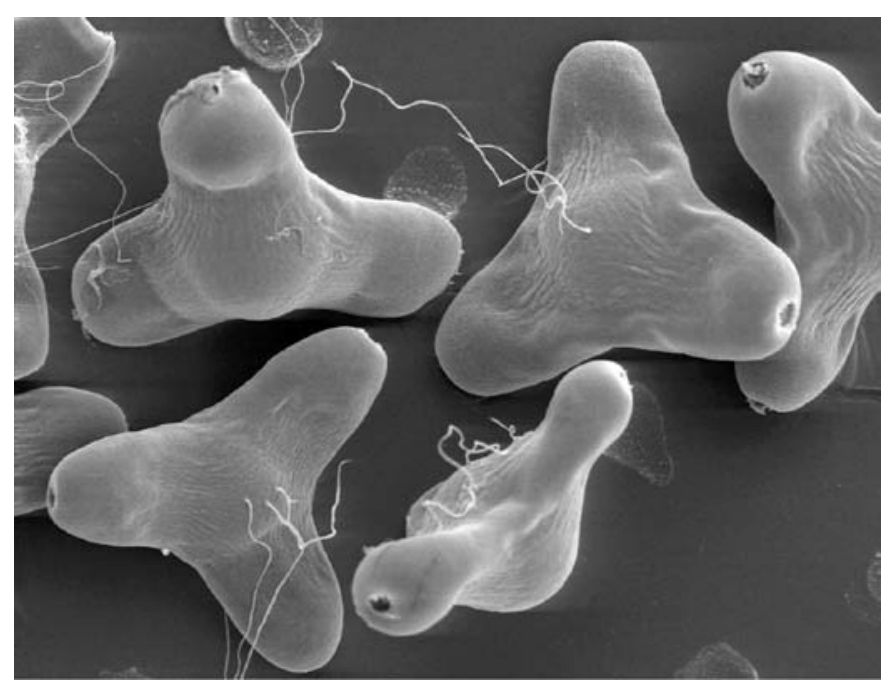

gleichzeitig die Th2-Antwort reduziert. Wenn aber durch die geringere Häufigkeit von Bakterien oder Viren das Th1-System nur unzureichend stimuliert wird, soll das zu einer zu hohen Empfindlichkeit des Th2-Systems führen: Allergische Reaktionen wären die Folge. Die Erklärung befriedigt nicht ganz, denn die Zunahme dervon Th1 modulierten Autoimmunerkrankungen lässt sich mit ihr nicht erklären. Eine alternative Hypothese ist die, dass es dem Immunsystem durch den Mangel an Mikroben an Reizen fehlt, sich richtig entwickeln zu können.

\section{Schutzfaktoren vor Allergenen gesucht}

Der Einfluss der Lebensweise und der Umwelt auf die Entstehung von Allergien war Thema einer ganzen Reihe von Forschungsprojekten. In den letzten zehn Jahren sind in Europa mehr als 15 Studien durchgeführt worden, die sich vor allem mit dem Einfluss des Landlebens auf das Vorkommen von Allergien beschäftigen. In allen Untersuchungen wurden Hinweise gefunden, dass das Leben auf dem Bauernhof vor Allergien schützt. Kinder, die im Kontakt mit Stalltieren aufwachsen, haben weniger häufig Allergien oder atopische Ekzeme. Beim Asthma ist der Zusammenhang weniger deutlich. Für einige Experten ist das ein Hinweis auf die Richtigkeit der Hygiene-Hypothese: Bauernkinder sind häufiger Bakterien, Viren oder Pilzen ausgesetzt als Stadtkinder. Einige Substanzen, wie z.B. etwa Endotoxin, das von Gram-negativen Bakterien stammt, sind in der Umgebung von Bauernkindern häufiger nachgewiesen worden als in der von Stadtkindern. Im Forschungsprojekt «Alex» haben Wissenschafter aus Österreich, Deutschland und der Schweiz zusammengearbeitet. Sie konnten zeigen, dass die Endotoxin-Konzentration in Matratzen von Kindern einen Einfluss auf die Häufigkeit von Heu- 
schnupfen, Asthma oder atopischen Reaktionen hat: Je höher sie ist, desto seltener sind die Allergien. Auch das Vorhandensein von Schimmelpilzen in der Umwelt von Kindern, die auf dem Bauernhof aufwachsen, scheint einen Schutzeffekt zu haben. Spuren von Schimmelpilzen wie Penicillium oder Aspergillus sind in Bauernhaushalten verbreiteter als in anderen.

Allerdings reichen die verschiedenen Mikroben-Parameter nicht aus, den Schutzeffekt des Landlebens vor Allergien ganz zu erklären. Es erscheint wahrscheinlich, dass es noch weitere mikrobielle Faktoren gibt, die das Immunsystem vor einem Überschiessen beim Kontakt mit einem harmlosen Allergen schützen. «Was es ist, das die Stallluft gesünder macht, wissen wir nicht», sagt von Mutius.

\section{Schutzfaktor Milch}

In der Zwischenzeit sind Hinweise aufgetaucht, dass es vor allem die industriell verarbeitete Milch sein könnte, die für die Zunahme der Allergien verantwortlich gemacht werden kann. «Ihr scheinen bestimmte Faktoren zu fehlen, die früher gegen Allergien geschützt haben», sagt Erika von Mutius. Milch spielt eine Schlüsselrolle beim Schutz vor Allergien und Asthma. Mit ihrer Wirkung haben sich Charlotte Braun-Fahrländer vom Institut für Sozial- und Präventivmedizin der Universität Basel und ihre Mitarbeiter auseinandergesetzt. Heute steht fest, dass nicht pasteurisierte Milch Kinder vor Allergien schützt. Unbekannt ist aber immer noch, weshalb es zu dieser Schutzwirkung kommt und welche molekularen oder immunologischen Mechanismen dabei eine Rolle spielen. In der Diskussion sind natürliche Inhaltsstoffe der Milch, aber ebenso auch mikrobielle Bestandteile aus Verunreinigungen. "Was auf immunologischer Ebene dort geschieht, wissen wir nicht», betont Erika von Mutius. «Wie die Wirkungskette zwischen Ursache und Allergie aussieht, ist vollkommen unklar», sagt von Mutius.

In einer internationalen Studie wurde deshalb die Schutzwirkung von Milchprodukten, Eiern, Fleisch und Gemüse epidemiologisch untersucht. Wenn die Milchprodukte direkt vom Bauernhof stammen und noch «naturbelassen» sind, scheinen sie tatsächlich vor Allergien zu schützen.

\section{Weniger Allergien}

Kinder, die während ihrer frühen Kindheit Milchprodukte direkt vom Bauernhof anstelle der industriell verarbeiteten aus dem Laden getrunken oder gegessen hatten, hatten deutlich weniger Allergien. Die frische Milch scheint vor allergischer Rhinokonjunktivitis, vor Asthma und vor atopischen Reaktionen zu schützen.

Diese Ergebnisse lassen den positiven Einfluss des Landlebens auf den Schutz vor Allergien in einem neuen Licht erscheinen. Sie zeigen, dass egal ist, wo Kinder aufwachsen, um einen funktionierenden Schutz vor Allergien $\mathrm{zu}$ haben. Voraussetzung ist, dass sie vom ersten Lebensjahr an frische, nicht pasteurisierte Milch vom Bauernhof trinken. Andere Faktoren wie etwa die besonders hohe Konzentration von Mikroben in Ställen oder Bauernhäusern spielen beim Schutz vor Allergien scheinbar keine wichtige Rolle.

Ähnliche Befunde zeigte auch eine Reihe anderer Untersuchungen in Deutschland, Grossbritannien oder Griechenland. Im Rahmen des Forschungsprogramms «Gabriel» sind mehr als 100000 Familien in ländlichen Gegenden Deutschlands, Österreichs, Polens und der Schweiz befragt worden. Die genetische Konstitution von mehr als 10000 Kindern, die auf Bauernhöfen oder in der Stadt aufwachsen, ist dabei untersucht worden. Ebenso wurden Proben der Milch genommen, die in den verschiedenen Gegenden getrunken wird.

\section{Schweizer Forscher mit dabei}

An den europäischen Forschungsprogrammen sind Wissenschafter aus der Schweiz federführend beteiligt. Am Institut für Sozial- und Präventivmedizin der Universität Basel ist die epidemiologische Allergieforschung seit Jahren ein wichtiger Schwerpunkt. Informationen über die Programme zur Erforschung der Allergie finden sich auf den Internetseiten der Projekte:

www.efraim-online.com

www.gabriel-fp6.org

$w w w$. forallvent.info

\section{Chance für die Prävention}

Analysen von frischer Milch zeigen, dass sie einen höheren Gehalt an Fett, Proteinen, Lactoferrin und somatischen Zellen aufweist als industriell verarbeitete, homogenisierte oder pasteurisierte Milch. Ob diese Bestandteile etwas mit der Schutzwirkung zu tun haben, ist unbekannt. Zumindest gibt es Hinweise, dass solche Substanzen, die mit dem Immunrezeptor CD14 interagieren, beim Schutz vor Allergien eine wichtige Rolle spielen könnten. Bei Menschen mit Veränderungen im CD14-Immunrezeptor ist die Schutzwirkung der Milch schwächer.

Sollte es im Laufe der Forschungsarbeiten möglich sein, die molekularen Faktoren identifizieren zu können, die für den Allergieschutz verantwortlich sind, könnte sich eine einfache Strategie zur Allergieprävention anbieten. Die Substanzen könnten der pasteurisierten Milch nach der Bearbeitung wieder zugesetzt werden, die dann die gleiche Schutzwirkung hätte wie die frische Milch direkt vom Bauern. «Das wäre in etwa mit der Fluorierung des Kochsalzes zur Kariesprophylaxe vergleichbar», sagt von Mutius. (rfi) 